\title{
Co-localization of zinc transporter 3 (ZnT3) with sensory neuromediators and/or neuromodulators in the enteric nervous system of the porcine esophagus
}

\author{
Joanna Wojtkiewicz $\cdot$ Liliana Rytel $\cdot$ Krystyna Makowska $\cdot$ Sławomir Gonkowski
}

Received: 14 February 2017 / Accepted: 29 March 2017/Published online: 17 April 2017

(C) The Author(s) 2017. This article is an open access publication

\begin{abstract}
Zinc transporter 3 (ZnT3) is one of the zinc transporters family. It is closely connected to the nervous system, where enables the transport of zinc ions from the cytoplasm to synaptic vesicles. This substance has been described within the central and peripheral nervous system, especially in the enteric nervous system (ENS). The aim of the present study was to describe the co-localization of ZnT3 with selected neuromediators and/or neuromodulators participating in sensory stimuli conduction in neurons of
\end{abstract}

J. Wojtkiewicz $(\bowtie)$

Department of Pathophysiology, Faculty of Medical

Sciences, University of Warmia and Mazury, Olsztyn, Poland

e-mail: joanna.wojtkiewicz@uwm.edu.pl

J. Wojtkiewicz

Laboratory for Regenerative Medicine, Faculty of

Medical Sciences, University of Warmia and Mazury,

Olsztyn, Poland

J. Wojtkiewicz

Foundation for the Nerve Cells Regeneration,

Warszawska Str. 30, Mazury, 10-082 Olsztyn, Poland

L. Rytel

Department of Internal Medicine and Clinic, Faculty of Veterinary Medicine, University of Warmia and Mazury, Oczapowskiego Str 15, 10-718 Olsztyn, Poland

K. Makowska $\cdot$ S. Gonkowski

Department of Clinical Physiology, Faculty of Veterinary

Medicine, University of Warmia and Mazury,

Oczapowskiego Str. 13, 10-718 Olsztyn, Poland the ENS within the porcine esophagus. Co-localization of ZnT3 with substance P (SP), leucine enkephalin (LENK) and calcitonin gene-related peptide (CGRP) was studied using standard double-immunofluorescence technique. The obtained results show that ZnT3, SP and/or LENK may occur in the same enteric neurons, and the degree of co-localization of these substances clearly depends on the fragment of esophagus studied and the type of enteric ganglia. In contrast, the co-localization of ZnT3 with CGRP was not observed during the present investigation. The obtained results suggest that ZnT3 in the ENS may be involved in the conduction of sensory and/or pain stimuli.

Keywords Enteric nervous system $\cdot$ Zinc transporters · Esophagus - Immunofluorescence technique $\cdot$ Pig

\section{Introduction}

It is widely known that zinc ( $\mathrm{Zn}$ ) is one of the most important microelements in the living organism. This metal, as a key component of many proteins and cofactor of over 300 enzymes, takes part in a wide range of processes connected with cellular metabolism, including, among others, DNA synthesis, cells division and differentiation, communication between cells and immunological processes (Vallee and Auld 1990; 
Vallee and Falchuk 1993; Takeda 2000). Zinc ions cannot cross the biological membranes by passive diffusion, and zinc homeostasis, which is essential for correct functioning of the organism, is maintained by metallothioneins (MTs) and transmembrane transporters (Hojyo and Fukada 2016; Hara et al. 2017).

Zinc transporters are divided into two groups: the solute-linked carrier 30 (SLC 30) protein family of zinc transporters (marked in the mammals from ZnT1 to ZnT10) and the Zip (Zrt- and Irt-like proteins) family (solute-linked carrier 39-SLC39) (Hara et al. 2017). First of them take place in the transport of zinc ions from cytoplasm into the lumen of organelles or extracellular space. In turn, Zip proteins have the opposite activity and transport zinc ions from extracellular space to the organelles (Eide 2006).

From all zinc transporters, only ZnT3 seems to be so closely linked to the nervous system, where it takes part in the transport of zinc ions from the cytoplasm to synaptic vesicles. Till now ZnT3 has been mainly described within the central nervous system (Frederickson et al. 2000, 2005), where it may be involved in sensory stimuli conduction, secretory activity and/or inhibitory processes (Frederickson et al. 2005). Moreover, it is known that ZnT3 plays some functions in adaptive phenomena under pathological factors, including epilepsy, mechanical injury or ischemia (Chi et al. 2008).

Contrary to the central nervous system, the knowledge concerning distribution and functions of ZnT3 in the peripheral nervous system is very scanty (Wang et al. 2003). In the light of the previous studies, it seems that the enteric nervous system (ENS) is the part of peripheral nervous system, where ZnT3 is present in relatively high amount (Gonkowski et al. 2009; Wojtkiewicz et al. 2012a, b, c, 2016).

The ENS, located in the wall of the gastrointestinal (GI) tract, due to the high degree of independence from the brain, a large number of neurons and complex structure, is often called "the second brain". The anatomy of the ENS depends on the fragment of the digestive tract and animal species, and in the esophagus it consists of two types of intramural ganglia: myenteric ganglia (MG) placed between the longitudinal and circular muscle layers and submucous ganglia (SG) - located near the lamina propria of the mucosa (Wojtkiewicz et al. 2016), which are interconnected with dense network of nerves. Enteric neurons control all functions of the GI tract both under physiological conditions and during pathological factors, including, among others, intestinal motility, excretive activity, mesenteric blood flow and immunological processes (Vasina et al. 2006). Cells of the ENS exhibit significant differentiation in terms of morphology, functions and electrophysiological properties, but the main criterion of enteric neurons classification is the "neurochemical coding", in the other words, the capability to expression of specific active substances (Furness 2012; Furness et al. 2014).

Several dozen factors, which most frequently may play functions of neuromediators and/or neuromodulators, have been described in the ENS. One of them is Znt3, that has been studied in the small and large intestine of human and pigs (Gonkowski et al. 2009; Wojtkiewicz et al. 2012a, b, c). It has been also studies in the esophagus (Wojtkiewicz et al. 2016), but till now the participation of $\mathrm{ZnT} 3$ in sensory stimuli conduction within this part of the GI tract has not been investigated at all. So, the aim of the present study was to describe the co-localization of ZnT3 with substance P (SP), leucine enkephalin (LENK) and calcitonin generelated peptide (CGRP), which are known as important factors involved in sensory stimuli conduction.

\section{Materials and methods}

The present study was made on six immature sows of the Large White Polish breed at the age of 8 weeks and about $18-20 \mathrm{~kg}$ body weight. Pigs were kept in typical laboratory conditions adapted for this animal species. The experiment was performed incompliance with the instructions of the Local Ethical Committee for the Experiments on Animals in Olsztyn (Poland) decision number.

After 3 days of adaptive period pigs were premedicated with Stressnil (Janssen, Belgium, $75 \mu \mathrm{l} / \mathrm{kg}$ of body weight, i.m.), after about $30 \mathrm{~min}$. euthanized using an overdose of sodium thiopental (Thiopental, Sandoz, Kundl-Rakúsko, Austria, i.v.) and perfused transcardially with $4 \%$ buffered paraformaldehyde. The fragments of cervical, thoracic and abdominal esophagus were collected from all sows. Tissues were post-fixed in the same paraformaldehyde solution, rinsed in phosphate buffer for three days and kept in $18 \%$ sucrose at $4{ }^{\circ} \mathrm{C}$. After at least two weeks the fragments of esophagus were frozen at $-23{ }^{\circ} \mathrm{C}$ and cut into $10 \mu \mathrm{m}$-thick sections using microtome (Microm, 
HM 525, Walldorf, Germany). The sections were subjected to routine double-labeling immunofluorescence technique according to method described previously by Gonkowski et al. (2013). This method in short consisted on the following stages (all actions were performed at room temperature): drying for $45 \mathrm{~min}$; incubation with a blocking solution, which included $10 \%$ normal goat serum, $0.1 \%$ bovine serum albumin, $0.01 \% \mathrm{NaN}_{3}$, Triton $\times-100$ and thimerozal in PBS for $1 \mathrm{~h}$; overnight incubation with a mixture of two "primary" antibodies raised in different species and directed towards zinc transporter 3 and one of the aftermentioned substances e.i. substance P, CGRP or LENK; incubation (for $1 \mathrm{~h}$ ) with species-specific antisera conjugated to FITC or biotin, which was visualized by a streptavidin-CY3 complex (the specification of primary and secondary antibodies used in the present study is shown in Table 1). The rinsing with PBS $(3 \times 10 \mathrm{~min}, \mathrm{pH} 7.4)$ was performed between the particular stages.

During the present investigation the standard controls of specificity of "primary" antibodies were performed. These included pre-absorption of the particular antisera with appropriate antigens, as well as "omission" and "replacement" tests and completely eliminated immunofluorescence signals.

Two methods of the evaluation of co-localization of ZnT3 with other substances studied were used. First of them consisted of the determination what percentage of all ZnT3-LI enteric neurons were cells immunoreactive to other substances studied. To this end at least 500 ZnT3-positive cell bodies in particular types of enteric ganglia were examined for immunoreactivity to the particular neuronal factors investigated, and ZnT3positive neurons were considered as representing $100 \%$ for all combinations. The second method was to determine what percentage of particular neuronal population (immunoreactive to SP, LENK or CGRP) were ZnT3-positive neurons. In this case at least neurons immunoreactive to SP, LENK or CGRP were evaluated for ZnT3-like immunoreactivity, and the numbers of cells immunoreactive to particular substances studied (SP, LENK or CGRP) were considered as $100 \%$.

Double-labeled perikarya (only neurons with clearlyvisible nucleus were included) were determined under an Olympus BX51 microscope equipped with epi-fluorescence and appropriate filter sets. The obtained results were pooled and presented as mean \pm SEM. To prevent double counting of the same neuronal cells, the sections evaluated during the present study were located at least $100 \mu \mathrm{m}$ apart. Statistical analysis was carried out with Student's $t$ test (Graphpad Prism v. 6.0; GraphPad Software Inc., San Diego, CA, USA). The differences were considered statistically significant at $\mathrm{p} \leq 0.05$.

\section{Results}

During this study the presence of ZnT3-positive neurons were observed in both types of enteric ganglia

Table 1 Specification of immune reagents used in the study: ZnT3 zinc transporter 3, $S P$ substance P, LENK leucine enkephalin, $C G R P$ calcitonin-gene related peptide, FITC fluorescein isothiocyanate, $C Y 3$ indocarbocyanine, $H$ heavy chain, $L$ light chain

\begin{tabular}{|c|c|c|c|c|c|c|}
\hline Antisera & Code & Host species & Dilution & \multicolumn{3}{|l|}{ Supplier } \\
\hline \multicolumn{7}{|c|}{ Primary antibody } \\
\hline ZnT3 & - & Rabbit & $1: 600$ & \multicolumn{3}{|c|}{ Gift from prof. Palmiter, University of Washington, Seattle, WA, USA } \\
\hline SP & $8450-0505$ & Rat & $1: 300$ & \multicolumn{3}{|c|}{ Biogenesis Inc., Poole, UK; http://www.biogenesis.co.uk } \\
\hline LENK & $4140-0355$ & Mouse & $1: 1000$ & \multicolumn{3}{|c|}{ Biogenesis Inc } \\
\hline CGRP & $\mathrm{T}-5027$ & Guinea pig & $1: 1000$ & \multicolumn{3}{|c|}{ Peninsula Labs., San Carlos, CA, USA; see Bachem AG; http://www.bachem.com } \\
\hline \multicolumn{5}{|l|}{ Reagent } & Dilution & Supplier \\
\hline \multicolumn{7}{|c|}{ Secondary antibodies } \\
\hline \multicolumn{5}{|c|}{ FITC-conjugated donkey-anti-mouse $\operatorname{IgG}(\mathrm{H}+\mathrm{L})$} & $1: 800$ & Jackson, 715-095-151, West Grove, PA, USA \\
\hline \multicolumn{5}{|c|}{ FITC-conjugated donkey-anti-rat IgG $(\mathrm{H}+\mathrm{L})$} & $1: 800$ & Jackson, 712-095-153 \\
\hline \multicolumn{5}{|c|}{ FITC-conjugated donkey-anti-guinea pig $\operatorname{IgG}(\mathrm{H}+\mathrm{L})$} & $1: 1000$ & Jackson, 706-095-148 \\
\hline \multicolumn{5}{|c|}{ Biotinylated goat anti-rabbit IgG } & $1: 1000$ & DAKO, E 0432, Carpinteria, CA, USA \\
\hline \multicolumn{5}{|c|}{ CY3- conjugated Streptavidin } & $1: 9000$ & Jackson, 016-160-084 \\
\hline
\end{tabular}



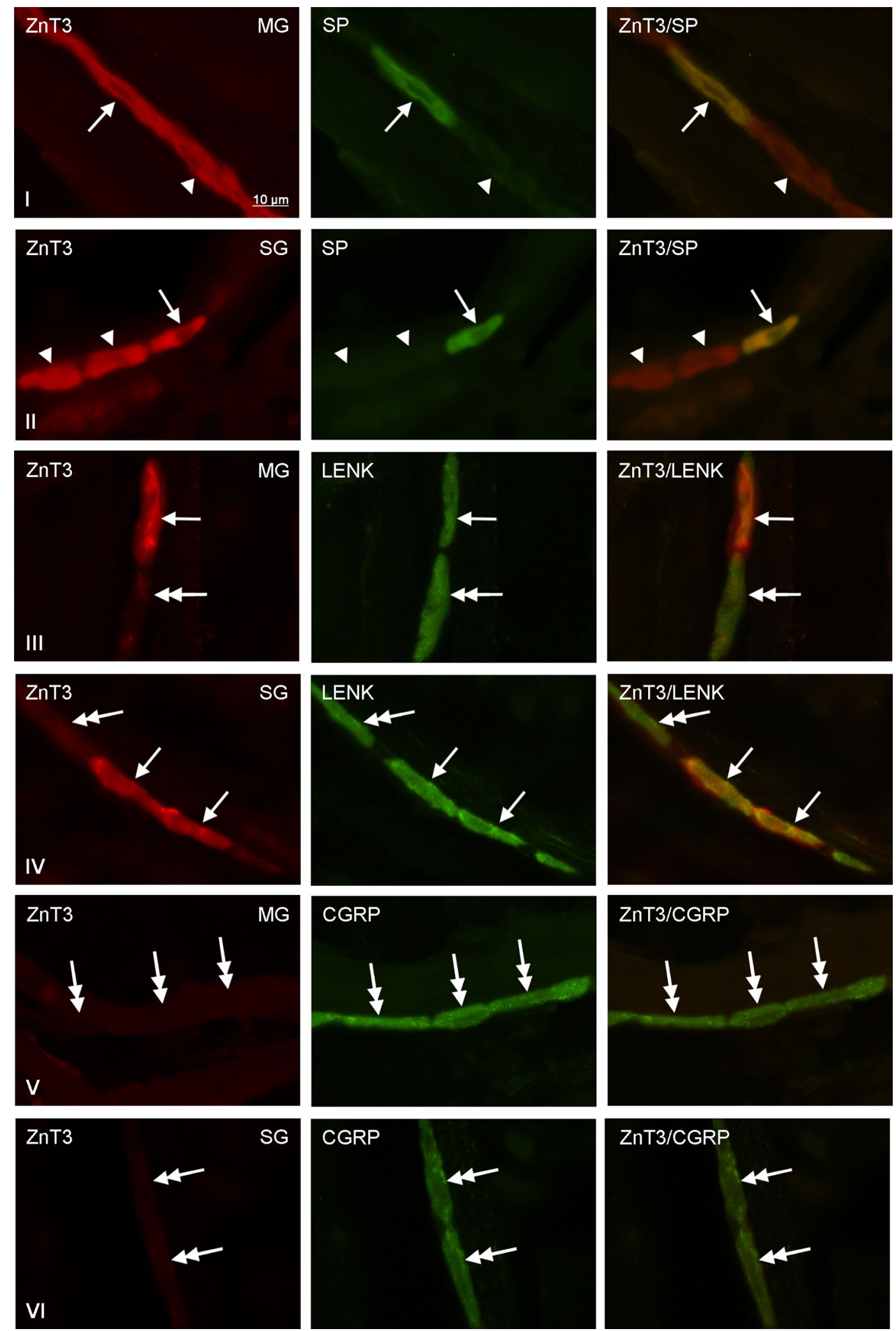

ZnT3/CGRP
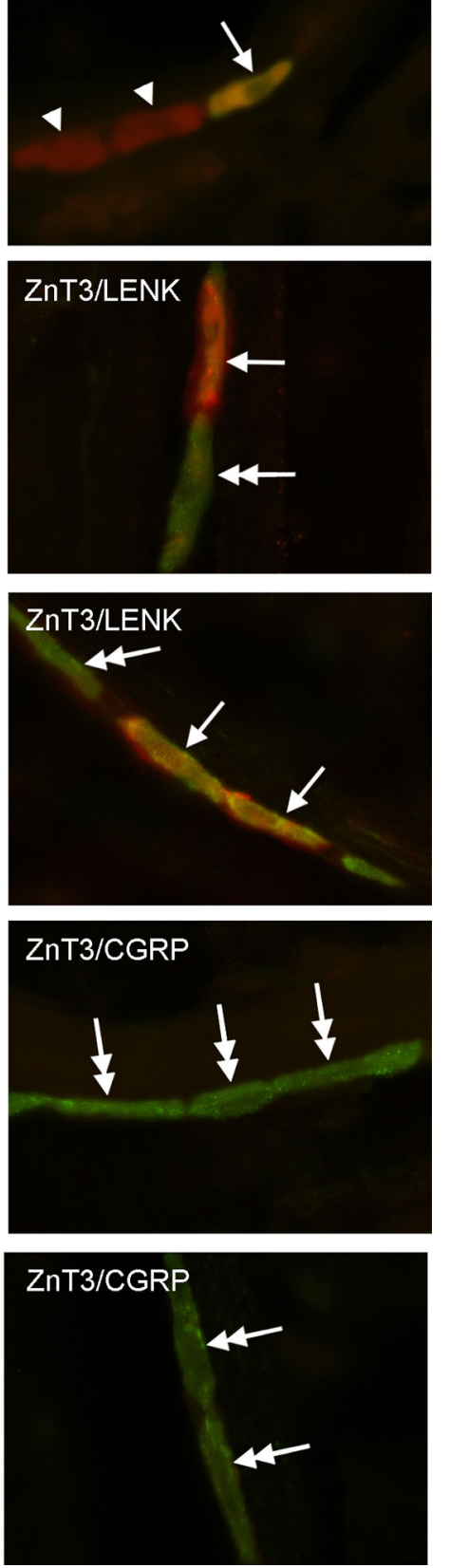
4Fig. 1 Co-localization of zinc transporter 3 (ZnT3) with sensory neuromediators and/or neuromodulators in the enteric nervous system within cervical part of the porcine esophagus: $M G$ myenteric ganglia, $S G$ submucous ganglia, $S P$ substance $\mathrm{P}$, LENK leucine enkephalin, CGRP calcitonin gene-related peptide. Neurons, where ZnT3 co-localizes with SP, LENK or CGRP are indicated with arrows. Neurons immunoreactive to ZnT3, but negative to SP, LENK or CGRP are indicated arrowheads. SP-, LENK- or CGRP-like immunoreactive neurons, where ZnT3 is not present are indicated with doubleheaded arrows. The right column of the pictures shows the overlap of both stainings

of all parts of esophagus studied, and this zinc transporter co-localized in the same cells with two from among three substances studied.

Co-localization of ZnT3 with SP and/or LENK were noted in the myenteric and submucous ganglia in cervical (Fig. 1), thoracic (Fig. 2) and abdominal (Fig. 3) esophagus, and the degree of its clearly depended on both the type of enteric ganglia and esophageal fragment (Tables 2,3). Contrary to SP and LENK, during the present investigation CGRP was not observed at all in esophageal enteric neurons immunoreactive to ZnT3 (Tables 2, 3), although on the other hand CGRP_-positive enteric neuronal cells (ZnT3-negative) were observed in the esophagus (Fig. 1V, VI; 2 V, VI; 3 V, VI).

In the cervical esophagus (Fig. 1) the significant differences in the degree of co-localization of $\mathrm{ZnT} 3$ with LENK were observed between myenteric and submucous ganglia. In the MG LENK was present within $80.3 \pm 3.2 \%$ of all ZnT3-positive cells, whereas in the SG the percentage of $\mathrm{ZnT} 3+$ l LENK + cells in relation to all neurons immunoreactive to ZnT3 amounted to only $21.5 \pm 7.7 \%$ (Table 2 ). Considerable lower percentage of ZnT3-positive neurons in the wall of cervical esophagus showed simultaneously the presence of SP. These values amounted to $4.4 \pm 1.8$ and $1.1 \pm 0.5 \%$ of all $\mathrm{ZnT} 3+$ neurons in the MG and SG, respectively (Table 2).

In the myenteric ganglia of thoracic esophagus (Fig. 2) the degree of co-localization of ZnT3 with LENK was clearly lower than in cervical part of this organ and achieved only $10.3 \pm 7.0 \%$ of all cells immunoreactive to $\mathrm{ZnT}$. In turn, within SG the presence of LENK was noted in $20.0 \pm 1.3 \%$ of ZnT3+ cells and this value was similar to those observed in the SG of the cervical esophagus (Table 2). The percentage of $\mathrm{ZnT} 3+/ \mathrm{SP}+$ neurons in relation to all Znt3-LI cells in thoracic esophagus was levelised in both types of enteric ganglia and significantly higher than in the cervical esophagus. These values amounted to $21.1 \pm 2.0 \%$ and $25.0 \pm 1$, 5 in the MG and SG, respectively (Table 2).

In the MG of abdominal esophagus (Fig. 3) the percentages of $\mathrm{ZnT} 3+/ \mathrm{LENK}+$ and $\mathrm{ZnT3}+/$ $\mathrm{SP}+$ neurons in relation to all cells immunoreactive to $\mathrm{ZnT} 3$ were levelised and achieved $16.6 \pm 4.7$ and $15.0 \pm 2.0 \%$, respectively. In the SG of abdominal esophagus the number of ZnT3-positive cells, which simultaneously immunopositive to LENK amounted to $22.0 \pm 6.4 \%$ and was similar to values observed in the cervical and thoracic esophagus. In turn the percentage of $\mathrm{ZnT} 3+/ \mathrm{SP}+$ neurons in relation to all Znt3-LI cells achieved $13.8 \pm 8.2 \%$ and was about $50 \%$ lower than in the thoracic esophagus, but significantly higher than within the cervical part of this organ (Table 2).

The degree of percentage of ZnT3-positive cells in relation to all population of neurons immunoreactive to LENK or SP also depended on the fragment of esophagus and type of enteric ganglion (Table 3 ). In the cervical esophagus (Fig. 1) both in the MG and SG relatively large number of LENK-LI cells showed also the presence of $\mathrm{ZnT3}$. These values amounted to $88.7 \pm 3.0$ and $72.0 \pm 5.8 \%$ in the MG and SG, respectively. The percentage of $\mathrm{ZnT} 3+$ neurons in relation to the population of cells immunoreactive to LENK in both types of enteric ganglia at first clearly decreased in caudal direction, reaching in the thoracic esophagus $75.0 \pm 3.0 \%$ in the MG and $50.0 \pm 4.0 \%$ in the SG before increasing again to $93.5 \pm 4.7 \%$ (in the $\mathrm{MG}$ ) and $91.7 \pm 3.0 \%$ (in the $\mathrm{SG}$ ) within the abdominal part of this organ (Table 3).

In the cervical esophagus the percentage of $\mathrm{ZnT} 3+$ cells in relation to all $\mathrm{SP}$-positive neurons amounted to $44.3 \pm 5.6 \%$ in the $\mathrm{MG}$ and $55.0 \pm 4.0 \%$ in the SG. In the MG of other part of esophagus studied these values clearly increased, especially in the thoracic esophagus, where all SPpositive cells observed during present investigations were also immunoreactive to ZnT3 (Table 3). In the MG of the abdominal esophagus the number of SP+l $\mathrm{ZnT} 3+$ cells in relation to all $\mathrm{SP}$ - positive neurons $(88.4 \pm 3.2 \%)$ was lower than in the thoracic fragment, but twice higher than within the cervical esophagus (Table 3). I turn, the percentage of ZnT3LI cells in relation to all $\mathrm{SP}+$ neurons studied in the $\mathrm{SG}$ of thoracic esophagus amounted to $25.0 \pm 4.0 \%$, 

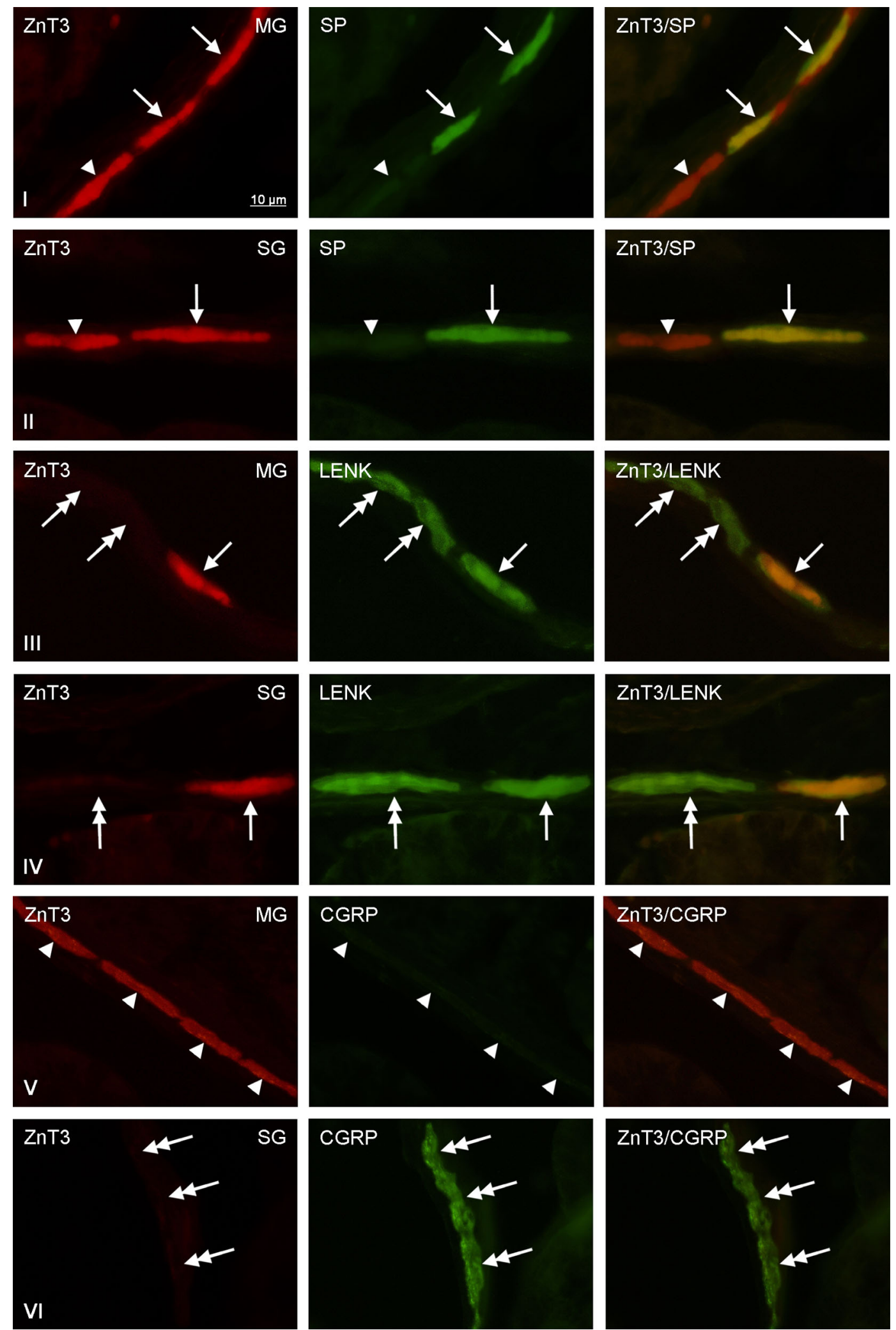
4Fig. 2 Co-localization of zinc transporter 3 (ZnT3) with sensory neuromediators and/or neuromodulators in the enteric nervous system within thoracic part of the porcine esophagus: $M G$ myenteric ganglia, $S G$ submucous ganglia, $S P$ substance $\mathrm{P}$, LENK leucine enkephalin, CGRP calcitonin gene-related peptide. Neurons, where ZnT3 co-localizes with SP, LENK or CGRP are indicated with arrows. Neurons immunoreactive to ZnT3, but negative to SP, LENK or CGRP are indicated arrowheads. SP-, LENK- or CGRP-like immunoreactive neurons, where ZnT3 is not present are indicated with doubleheaded arrows. The right column of the pictures shows the overlap of both stainings

and within abdominal esophagus achieved $63.3 \pm 8.2 \%$.

\section{Discussion}

The results obtained during this study show that $\mathrm{ZnT} 3$ is present in the enteric neurons of all fragments of esophagus studied, what is in agreement with previous investigations (Wojtkiewicz et al. 2016). It should be pointed out that exact functions of ZnT3 within enteric neurons still remain unclear and are often described by analogy with the central nervous system, where the activity of this zinc transporter is better known. In the brain Znt3 takes part in the regulation of zinc ions levels in neuronal cells (Palmiter et al. 1996; Palmiter and Huang 2004) and it seems to be a typical substance for neurons that used zinc as a neuromodulator (Takeda et al. 2013). Moreover ZnT3 facilitates the transport of zinc from the cytoplasm to synaptic vesicles (Palmiter et al. 1996), and thereby vicariously influences on the synaptic zinc ions levels. So ZnT3 seems to be a key factor, which is involved in the maintenance of synaptic $\mathrm{Zn}^{2+}$ homeostasis. On the other hand, it is relatively well established that this homeostasis is essential for correct functioning of the nervous system, because both excess and deficiency of synaptic zinc ions may cause disturbances in neuronal physiology and neurodegeneration (Weiss et al. 2000; Takeda et al. 2013). Probably, the involvement of $\mathrm{ZnT} 3$ in the regulation of synaptic $\mathrm{Zn} 2^{+}$levels is at the very heart of previously described changes in the expression of this zinc transporter during various pathological processes in the brain, including ischemia Huntington's, Alzheimer's and Parkinson's diseases, epilepsy and mechanical damage (Devirgiliis et al. 2007; Chi et al. 2008; Whitfield et al. 2015).
Moreover, it is known that $\mathrm{ZnT} 3$ in the central nervous system may take part in regulatory processes connected both with sensory stimuli conduction and secretory activity of neuronal cells (Danscher et al. 2001, 2003), and some previous studies view ZnT3 as the marker of inhibitory nerve fibers called zinc enriched nerves (ZEN) (Jo et al. 2000).

One could only conjecture that Znt3 in the enteric nervous system may play similar functions. Previous studies showed that this zinc transporter is present in various types of enteric neurons and co-localizes with a wide range of other neuronal substances (Wojtkiewicz et al. 2012b, c, 2016), what can suggest miscellaneous functions of this substance in the ENS. In turn, the results obtained in the present study, where co-localization of ZnT3 with LENK and/or SP was observed, can suggest involvement of this zinc transporter in sensory stimuli conduction within the gastrointestinal tract. Two facts enforces this thesis. Firstly, both SP and LENK are factors, that often are present in sensory neurons and their role in sensory and pain conduction is commonly known (De Schepper et al. 2004; Shimizu et al. 2008). Moreover, it is relatively established that substances co-localized in the same neuronal cells usually play similar functions (Wojtkiewicz et al. 2012a). Secondly, ZnT3 has been previously described as the factor, that can take part in sensory neurons activity within the central nervous system (Danscher et al. 2001).

On the other hand, it should be pointed out that the contribution of ZnT3 in sensory conduction in the ENS are not completely clear. Neither in the esophagus (results of the present study), nor in other fragments of the gastrointestinal tract (Wojtkiewicz et al. 2012b, c) the co-localization of ZnT3 with CGRP has not been observed, and CGRP seems to be one of the main sensory neurofactors in the ENS and by some investigators is considered to be the marker of the intrinsic primary afferent neurons-enteric sensory neurons and afferent component the short, intestinal intramural reflex arcs (Mawe and Sharkey 2016).

Absence of the co-localization of ZnT3 and CGRP may suggest on the one hand that CGRP is not the marker of intrinsic primary afferent neurons in the pig, and on the other hand that the co-localization observed during the present study affects not only sensory neurons. It is all the more probably because LENK and $\mathrm{SP}$, apart from commonly known involvement in the sensory conduction, may play other various roles in 

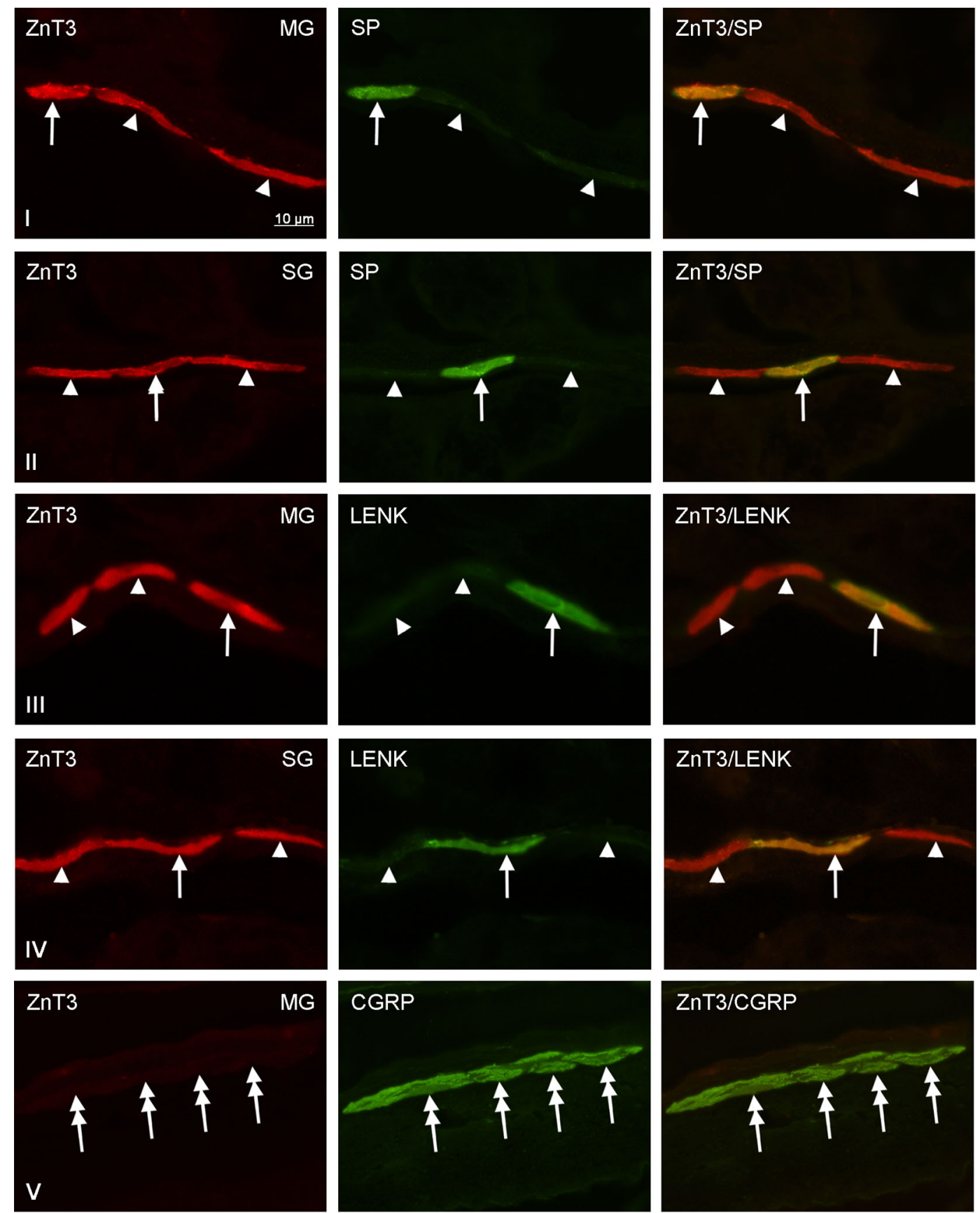

ZnT3/LENK
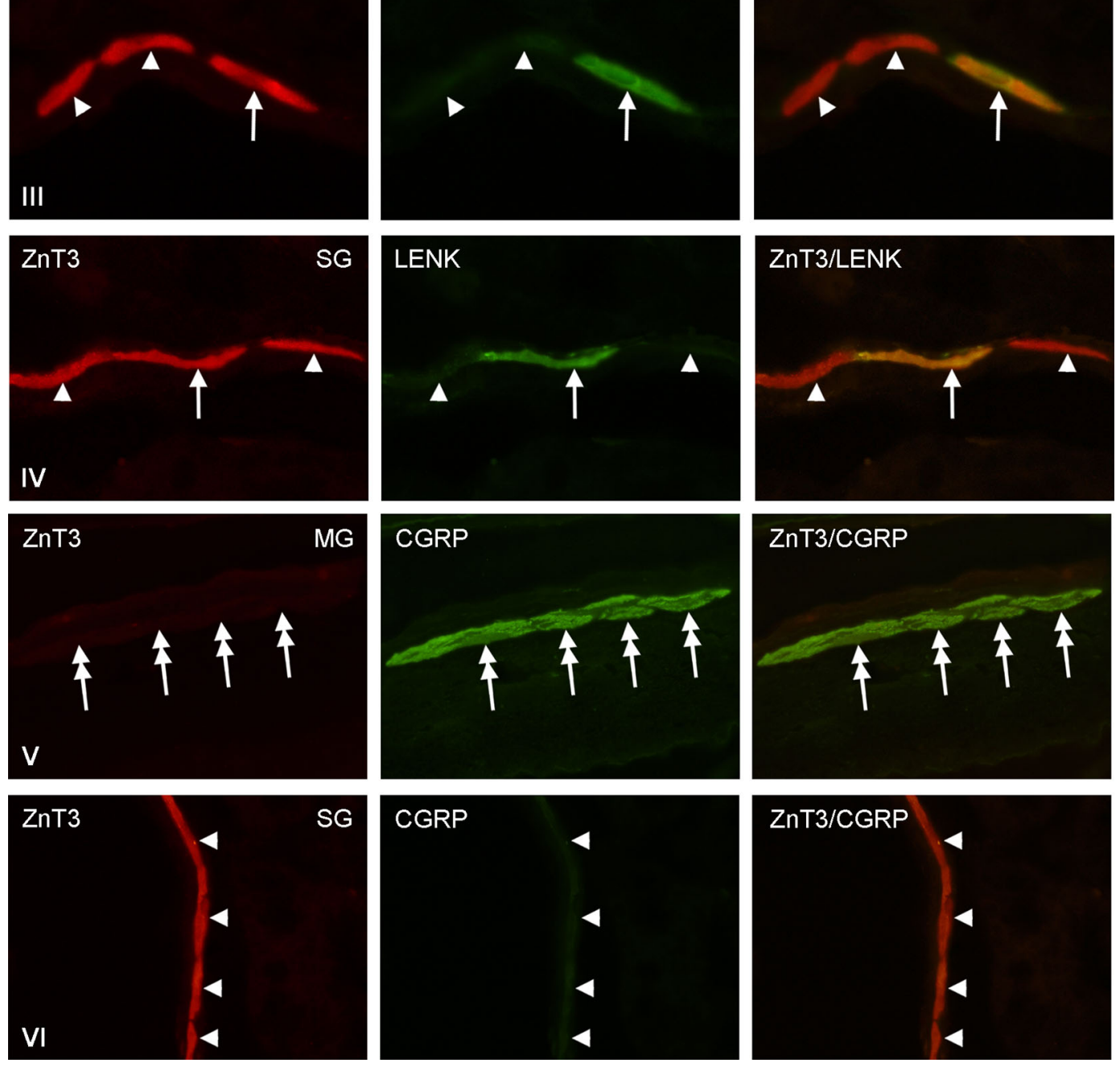
4Fig. 3 Co-localization of zinc transporter 3 (ZnT3) with sensory neuromediators and/or neuromodulators in the enteric nervous system within abdominal part of the porcine esophagus: $M G$ myenteric ganglia, $S G$ submucous ganglia, $S P$ substance $\mathrm{P}$, LENK leucine enkephalin, CGRP calcitonin gene-related peptide. Neurons, where ZnT3 co-localizes with SP, LENK or CGRP are indicated with arrows. Neurons immunoreactive to ZnT3, but negative to SP, LENK or CGRP are indicated arrowheads. SP-, LENK- or CGRP-like immunoreactive neurons, where ZnT3 is not present are indicated with doubleheaded arrows. The right column of the pictures shows the overlap of both stainings

Table 2 The percentage (mean \pm SEM) of neurons immunoreactive to leucine enkephalin (LENK), substance $P$ (SP), and/or calcitonin gene-related peptide (CGRP) in relation to the population of ZnT3-positive neurons in the enteric nervous system of the porcine esophagus

\begin{tabular}{lll}
\hline & Myenteric ganglia & Submucous ganglia \\
\hline Cervical esophagus & & \\
ZnT3+/LENK+ & $80.3 \pm 3.2$ & $21.5 \pm 7.7$ \\
ZnT3+/SP+ & $4.4 \pm 1.8$ & $1.1 \pm 0.5$ \\
ZnT3+/CGRP+ & 0 & 0 \\
Thoracic esophagus & & \\
ZnT3+/LENK+ & $10.3 \pm 7.0$ & $20.0 \pm 1.3$ \\
ZnT3+/SP+ & $21.1 \pm 2.0$ & $25.0 \pm 1.5$ \\
ZnT3+/CGRP+ & 0 & 0 \\
Abdominal esophagus & \\
ZnT3+/LENK+ & $93.5 \pm 3.8$ & $91.7 \pm 3.0$ \\
ZnT3+/SP+ & $15.0 \pm 2.0$ & $13.8 \pm 8.2$ \\
ZnT3+/CGRP+ & 0 & 0 \\
\hline
\end{tabular}

ZnT3 positive neurons were considered as representing $100 \%$

the ENS. Namely, SP influences the intestinal motility, and the character of this activity depends on animal species and the fragment of the digestive tract. For example SP strongly induces intestinal muscles contractions in rats and dogs (Lördal et al. 1993; Thor et al. 1982), contrary to the human, where this activity of SP is rather infinitesimal (Lördal et al. 1997). Moreover, SP in the ENS also regulates the excretive activity of the digestive tract and mesenteric blood flow (Brunsson et al. 1995), as well as takes part in adaptive and neuroprotective processes under pathological factors (Shimizu et al. 2008; Gonkowski 2013). In turn, LENK participates in inhibition of intestinal motility and secretion and takes part in immunological activity of the GI tract (Puig and Pol 1998; Pol et al. 2003; De Schepper et al. 2004).
Table 3 The percentage (mean \pm SEM) of zinc-transporter3-like immunoreactive perikarya in neuronal populations immunoreactive to particular active substances studied

\begin{tabular}{cll}
\hline & Myenteric ganglia & Submucous ganglia \\
\hline Cervical esophagus & & \\
LENK+/ZnT3+ & $88.7 \pm 3.0$ & $72.0 \pm 5.8$ \\
SP+/ZnT3+ & $44.3 \pm 5.6$ & $55.0 \pm 4.0$ \\
CGRP+/ZnT3+ & 0 & 0 \\
Thoracic esophagus & & \\
LENK+/ZnT3+ & $75.0 \pm 3.0$ & $50.0 \pm 4.0$ \\
SP+/ZnT3+ & 100 & $25.0 \pm 4.0$ \\
CGRP+/ZnT3+ & 0 & 0 \\
Abdominal esophagus & \\
LENK+/ZnT3+ & $16.6 \pm 4.7$ & $22.0 \pm 6.4$ \\
SP+/ZnT3+ & $88.4 \pm 3.2$ & $63.3 \pm 8.2$ \\
ZnT3+/CGRP+ & 0 & 0
\end{tabular}

The number of neurons immunoreactive to each substance were considered as representing $100 \%$

Moreover, the degree of co-localization of $\mathrm{ZnT3}$ with SP and/or LENK observed during the present study significantly differs from those observed in other parts of the porcine digestive tract (Wojtkiewicz et al. 2012b, c), what may suggest that exact roles of both ENS as a whole, as well as ZnT3 as an active substance clearly depend on the fragment of the digestive tract.

To sum up, the results obtained during the present study strongly suggest that ZnT3 in the ENS of the porcine esophagus may take part in the sensory and pain stimuli conductions. On the other hand some aspects connected with this role of $\mathrm{ZnT3}$ remain unclear and, due to complicated construction of the ENS, as well as omnidirectional activities of this zinc transporter, require the further investigations.

Open Access This article is distributed under the terms of the Creative Commons Attribution 4.0 International License (http:// creativecommons.org/licenses/by/4.0/), which permits unrestricted use, distribution, and reproduction in any medium, provided you give appropriate credit to the original author(s) and the source, provide a link to the Creative Commons license, and indicate if changes were made.

\section{References}

Brunsson I, Fahrenkrug J, Jodal M, Sjöqvist A, Lundgren O (1995) Substance P effects on blood flow, fluid transport 
and vasoactive intestinal polypeptide release in the feline small intestine. J Physiol 15:727-734

Chi ZH, Wang X, Cai JQ, Stoltenberg M, Danscher G, Wang ZY (2008) Zinc transporter 3 immunohistochemical tracing of sprouting mossy fibres. Neurochem Int 52:1305-1309. doi:10.1016/j.neuint.2008.02.008

Danscher G, Jo SM, Varea E, Wang Z, Cole TB, Schroder HD (2001) Inhibitory zinc-enriched terminals in mouse spinal cord. Neuroscience 105:941-947. doi:10.1016/S03064522(01)00243-3

Danscher G, Wang Z, Kim YK, Kim SJ, Sun Y, Jo SM (2003) Immunocytochemical localization of zinc transporter 3 in the ependyma of the mouse spinal cord. Neurosci Lett 342:81-84

De Schepper HU, Cremonini F, Park MI, Camilleri M (2004) Opioids and the gut: pharmacology and current clinical experience. Neurogastroenterol Motil 16:383-394. doi:10. 1111/j.1365-2982.2004.00513.x

Devirgiliis C, Zalewski PD, Perozzi G, Murgia C (2007) Zinc fluxes and zinc transporters genes in chronic diseases. Mutat Res 622:84-93. doi:10.1016/j.mrfmmm.2007.01. 013

Eide DJ (2006) Zinc transporters and the cellular trafficking of zinc. Biochim Biophys Acta 1763:711-722. doi:10.1016/j. bbamcr.2006.03.005

Frederickson CJ, Suh SW, Silva D, Frederickson CJ, Thompson RB (2000) Importance of zinc in the central nervous system: the zinc-containing neuron. J Nutr 130:1471S-1483S

Frederickson CJ, Koh JY, Bush AI (2005) The neurobiology of zinc in health and disease. Nat Rev Neurosci 6:449-462. doi:10.1038/nrn1671

Furness JB (2012) The enteric nervous system and neurogastroenterology. Nat Rev Gastroenterol Hepatol 9:286-294. doi:10.1038/nrgastro.2012.32

Furness JB, Callaghan BP, Rivera LR, Cho HJ (2014) The enteric nervous system and gastrointestinal innervation: integrated local and central control. Adv Exp Med Biol 817:39-71. doi:10.1007/978-1-4939-0897-4_3

Gonkowski S (2013) Substance P as a neuronal factor in the enteric nervous system of the porcine descending colon in physiological conditions and during selected pathogenic processes. BioFactors 39:542-551. doi:10.1002/biof.1097

Gonkowski S, Kaminska B, Landowski P, Skobowiat C, Burliński P, Majewski M, Calka J (2009) A population of zinc transporter 3-like immunoreactivite neurons is present in the ganglia of human descending colon. Adv Clin Exp Med 18:243-248

Gonkowski S, Kaminska B, Landowski P, Calka J (2013) Immunohistochemical distribution of cocaine- and amphetamine-regulated transcript peptide-like immunoreactive (CART-LI) nerve fibers and various degree of co-localization with other neuronal factors in the circular muscle layer of human descending colon. Histol Histopathol 28:851-858. doi:10.14670/HH-28.851

Hara T, Takeda TA, Takagishi T, Fukue K, Kambe T, Fukada T (2017) Physiological roles of zinc transporters: molecular and genetic importance in zinc homeostasis. J Physiol Sci. doi:10.1007/s12576-017-0521-4

Hojyo S, Fukada T (2016) Zinc transporters and signaling in physiology and pathogenesis. Arch Biochem Biophys 611:43-50. doi:10.1016/j.abb.2016.06.020
Jo SM, Won MH, Cole TB, Jansen MS, Palmiter RD, Danscher G (2000) Zinc-enriched (ZEN) terminals in mouse olfactory bulb. Brain Res 865:227-236

Lördal M, Johansson C, Hellström PM (1993) Myoelectric pattern and effects on small bowel transit induced by the tachykinins neurokinin A, neurokinin B, substance P and neuropedtide $\mathrm{K}$ in the rat. J Gastrointest Motil 5:33-40. doi:10.1111/j.1365-2982.1993.tb00105.x

Lördal M, Theodorsson E, Hellström PM (1997) Tachykinins influence interdigestive rhythm and contractile strength of human small intestine. Dig Dis Sci 42:1940-1949

Mawe GM, Sharkey KA (2016) The intrinsic reflex circuitry of the inflamed colon. Adv Exp Med Biol 891:153-157. doi:10.1007/978-3-319-27592-5_15

Palmiter RD, Huang L (2004) Efflux and compartmentalization of zinc by members of the SLC30 family of solute carriers. Pflugers Arch 447:744-751. doi:10.1007/s00424-003-1070-7

Palmiter RD, Cole TB, Quaife CJ, Findley SD (1996) ZnT-3, a putative transporter of zinc into synaptic vesicles. Proc Natl Acad Sci USA 93:14934-14939

Pol O, Palacio JR, Puig MM (2003) The expression of delta- and kappa-opioid receptor is enhanced during intestinal inflammation in mice. J Pharmacol Exp Ther 306:455-462. doi:10.1124/jpet.103.049346

Puig MM, Pol O (1998) Peripheral effects of opioids in a model of chronic intestinal inflammation in mice. J Pharmacol Exp Ther 287:1068-1075

Shimizu Y, Matsuyama H, Shina T, Takewaki T, Furness JB (2008) Tachykinins and their functions in the gastrointestinal tract. Cell Mol Life Sci 65:295-311. doi:10.1007/ s00018-007-7148-1

Takeda A (2000) Movement of zinc and its functional significance in the brain. Brain Res Rev 34:137-148

Takeda A, Nakamura M, Fujii H, Tamano H (2013) Synaptic $\mathrm{Zn} 2+$ homeostasis and its significance. Metallomics 5:417-423. doi:10.1039/C3MT20269K

Thor PJ, Sendur R, Konturek SJ (1982) Influence of substance P on myoelectric activity of the small bowel. Am J Physiol 243:G493-G496

Vallee BL, Auld DS (1990) Zinc coordination, function and structure of zinc enzymes and other proteins. Biochemistry 29:5647-5659. doi:10.1021/bi00476a001

Vallee BL, Falchuk KH (1993) The biochemical basis of zinc physiology. Physiol Rev 73:79-118

Vasina V, Barbara G, Talamonti L, Stanghellini V, Corinaldesi R, Tonini M, De Ponti F, De Giorgio R (2006) Enteric neuroplasticity evoked by inflammation. Auton Neurosci 126-127:264-272. doi:10.1016/j.autneu.2006.02.025

Wang ZY, Danscher G, Dahlstrom A, Li JY (2003) Zinc transporter 3 and zinc ions in the rodent superior cervical ganglion neurons. Neuroscience 120:605-616. doi:10. 1016/S0306-4522(03)00419-6

Weiss JH, Sensi SL, Koh JY (2000) Zn2+: a novel ionic mediator of neural injury in brain disease. Trends Pharmacol Sci 21:395-401

Whitfield DR, Vallortigara J, Alghamdi A, Hortobágyi T, Ballard C, Thomas AJ, O'Brien JT, Aarsland D, Francis PT (2015) Depression and synaptic zinc regulation in Alzheimer disease, dementia with lewy bodies, and Parkinson disease dementia. Am J Geriatr Psychiatry 23:141-148. doi:10.1016/j.jagp.2014.05.001 
Wojtkiewicz J, Gonkowski S, Bladowski M, Majewski M (2012a) Characterisation of cocaine- and amphetamineregulated transcript-like immunoreactive (CART-LI) enteric neurons in the porcine small intestine. Acta Vet Hung 60:371-381

Wojtkiewicz J, Gonkowski S, Równiak M, Crayton R, Majewski M, Jałyński M (2012b) Neurochemical characterization of zinc transporter 3-like immunoreactive $(\mathrm{ZnT} 3(+))$ neurons in the intramural ganglia of the porcine duodenum. J Mol Neurosci 48:766-776. doi:10.1007/s12031-0129855-9
Wojtkiewicz J, Równiak M, Crayton R, Majewski M, Gonkowski S (2012c) Chemical coding of zinc-enriched neurons in the intramural ganglia of the porcine jejunum. Cell Tissue Res 350:215-223. doi:10.1007/s00441-012-1486-5

Wojtkiewicz J, Makowska K, Bejer-Olenska E, Gonkowski S (2016) Zinc transporter 3 (Znt3) as an active substance in the enteric nervous system of the porcine esophagus. J Mol Neurosci. doi:10.1007/s12031-016-0854-0 